Stearin-, Öl- und Linolsäure $(1: 1: 1: 1)$ zugesetzt. Bei Milchproben, die den Methylesterfleck schon ursprünglich zeigten, vergrößerte sich dieser Fleck sehr stark, während der der freien Fettsäuren nur in geringerem $\mathrm{Maße}$ verstärkt wurde (Abb. 1. IV.). Bei Milchproben hingegen, deren methanolische Lipidextrakte keinen Methylesterfleck aufwiesen, entwickelten sich auch nach Zusatz von Fettsäuren keine Methylesterflecke, oder es zeigten sich nur ganz geringe, kaum sichtbare Flecke. Demgegenüber vergrößerte sich auf dem Chromatogramm der Fleck der schon zu Beginn anwesenden freien Fettsäuren in beträchtlichem $\mathrm{Maße}$ (Abb. 1. V.). Hieraus entspringt die Vermutung, da $B$ in gewissen Fällen ein Faktor in der Frauenmilch gegenwärtig sei, der die Methylestersynthese aus den freien Fettsäuren fördert.
Die Fettsäurezusammensetzung der von der Schicht eluierten Artefekte wurde direkt gaschromatographisch bis $\mathrm{C}_{18}$ untersucht. (Gerät: Ärogtaph A-90-P; Kolonne: $10 \mathrm{FuB},{ }_{1}^{1} \mathrm{~A}^{1 "}$; Füllung: Chromosorb W 60-80 mesh, 15\% DEGS; Trägergas: $\mathrm{H}_{2}, 60 \mathrm{~m} /$ /Min.; Heiqstrom: $200 \mathrm{~mA}$; Temperatur: Kolonne $180^{\circ}$, Detektor $240^{\circ}$, Injektor $220^{\circ}$, Kollektor $215^{\circ}$ ).

Wie zu erwarten war, erschienen die Spitzen des Diagramms den Retentionszeiten der fettsauren Methylester entsprechend. In der Tabelle 1 ist auf Grund der "peak-area" der gaschromatographischen Fraktogramme das Verhältnis der Methylester der freien Fettsäuren in den ohne Methanol extrahierten Lipiden sowie der mit dem Methanolverfahren aus den Lipiden entstandenen Methylester-Artefakte einer repräsentativen Frauenmilchprobe dargestellt.

Tab. 1

\begin{tabular}{|c|c|c|c|c|c|c|c|c|}
\hline & \multicolumn{8}{|c|}{ Methylester \% } \\
\hline & $\mathrm{C}_{10}$ & $\mathrm{C}_{12}$ & $\mathrm{C}_{14}$ & $\mathrm{C}_{16}$ & $\mathrm{C}_{16} *$ & $\mathrm{C}_{18}$ & $\mathrm{C}_{18} *$ & $\mathrm{C}_{18} * *$ \\
\hline Methylester der freien Fettsäuren im Frauenmilchlipid & 7,2 & 3,9 & 2,4 & 8,2 & 1,0 & 9,5 & 40,2 & 27,6 \\
\hline Methylester-Artefakte im Frauenmilchlipid & 3,6 & 6,8 & 2,8 & 14,6 & 2,0 & 14,0 & 35,8 & 20,4 \\
\hline
\end{tabular}

* einfach ungesättigt

** doppelt ungesättigt

\title{
Literatur
}

1. Folch, J., I. Ascoli, M. Lees, J. A. Meath und F. N. Le Baron, J. biol. Chemistry 191, 833 (1951). - 2. Zöllner, N. und G. Wolfram, Klin. Wschr. 40, 1098 (1962). - 3. Bloor, W. R., J. biol. Chemistry, 77, 53 (1928). - 4. Kaufmann, H. P. und H. Garlofr, Fette, Seifen, einschließl. Anstrichmittel 62, 679 (1960). - 5. Pfeifer, J. J., Mikrochim. Acta 529 (1962). -
6. Mangold, H. K., Aliphatische Lipide in E. Stahl: Dünnschichtchromatographie, S. 158. Springer-Verlag, Berlin-GöttingenHeidelberg (1962). - 7. JákY, M., A. KoRÁNYI und P. HágonY, Növényolaj Kutató Int. Közl.S. 1., Budapest, Ungarisch (1962). 8. Medgyessy, G., persönliche Mitteilung.

Dr. G. Czeglédi-Jankó Institut für Ernährungswissenschảft Budapest IX., Gyáli ut 3/a

\section{Zur quantitativen Bestimmung von Indican in Urin und Serum}

\author{
Von D. Müring und H. J. Burgard
}

Aus der 1. Medizinischen Klinik der Universität des Saarlandes, Homburg/Saar (Direktor: Prof. Dr. med. F. Doenecke)

(Der Schriftleitung zugegangen am 14. Mai 1964)

\begin{abstract}
Auf dem Prinzip der Farbreaktion nach Jolles wurde eine Methode zur Bestimmung von Indican in Urin und Serum entwickelt, deren einfache Ausführung, deren Genauigkeit und Empfindlichkeit bei geringen Mengen an Untersuchungsmaterial eine Anwendung für klinische $Z$ wecke ermöglichen.

A method was developed for the determination of indican in urine and serum, based on the colour reaction of Jolles. Its simplicity of operation, accuracy and sensitivity with small amounts of experimental material make it suitable for clinical purposes.
\end{abstract}

Die bisher bekannten Methoden der Bestimmung von Harnindican (Schwefelsäure-mono-[indolyl-(3)-ester] = Indoxylschwefelsäure) für klinische Zwecke waren entweder zu ungenau und unempfindlich oder zu schwierig und zeitraubend, als daß sie sich in der Klinik durchgesetzt hätten. Die hier angestellten Untersuchungen gehen auf die Feststellung von Jolles (1) zurück, der Indoxyl und Thymol zu einem Farbkomplex oxydierte.
Dieser besteht nach seinen Untersuchungen mit großer Wahrscheinlichkeit aus [ $p$-Cymol-(2)]-[indol-(2)]-indolignon. Von den verschiedenen Modifikationen dieser Methode bringt diejenige von RAPPAPORT und ENGELBERG (2) einen neuen Gesichtspunkt: Es bildet sich bei Verwendung von Trichloressigsäure als Eiweißfällungsmittel ein Tropfen „Öl“, in dem die Farbe löslich ist. Dieses Verhalten wurde in der Methode von 
Shivaram und D. Mürrng (3), die dieser Methode zugrunde liegt, angewandt.

\section{Methode}

Indican wird unter der hydrolytischen Wirkung von konz. $\mathrm{HCl}$ in Indoxyl überführt, welches mit Thymol durch $\mathrm{FeCl}_{3}$ zu einem rotvioletten Farbstoff oxydiert wird. Gleichzeitig bilden sich zwei Phasen. In der unteren Phase konnte gaschromatographisch Trichloressigsäureäthylester nachgewiesen werden, in dem noch Alkohol und Trichloressigsäure gelöst sind. Der Farbstoff wird durch die untere Phase quantitativ extrahiert. Der Vorgang kann als "nascierende Extraktion" bezeichnet werden. Nach Absaugen des Úberstandes wird mit Methanol bis zu einer bestimmten Marke aufgefüllt und durch Schütteln eine klare Farblösung hergestellt. Diese wird photometriert.

Trotz der einfachen Ausführung und der geringen Ausgangsmengen bietet die Methode eine für klinische Zwecke voll ausreichende Empfindlichkeit und Genauigkeit. Die Untersuchungen der Reproduzierbarkeit und Spezifität zeigen die Brauchbarkeit der Methode für klinische Untersuchungen.

\section{Versuche}

\section{Bestimmung von Indican im Urin}

\section{Reagenzien}

a) 20-proz. wäßrige Trichloressigsäurelösung

b) 0,5-proz. Thymollösung in Athanol

c) Lösung: $0,3 \mathrm{~g} \mathrm{FeCl}_{3}, 50 \mathrm{~g}$ Trichloressigsäure, konz. $\mathrm{HCl}$ ad $100 \mathrm{ml}$

d) Methanol als Lösungsmittel

e) 0,1-proz. wäßrige Indicanlösung zur Darstellung der Eichkurve (Fluka).

\section{Ausführung}

$0,5 \mathrm{ml}$ eines 24-Stunden-Urins werden in ein graduiertes Zentrifugenröhrchen gegeben. Zeigt sich beim Hinzufügen von Lösung a) eine Trübung, so wird zunächst $1 \mathrm{~m} /$ dieser Lösung hinzugefügt und gut geschüttelt. Es wird zentrifugiert und in ein graduiertes Zentrifugenröhrchen abgegossen. Der Niederschlag wird in $1 \mathrm{~m} l$ der Lösung a) aufgeŝchwemmt und nochmals żentrifugiert. Der Uberstand wird dem ersten hinzugefügt. Zeigt der Urin beim Hinzufügen der Lösung a) keine Trübung, so werden sofort $2 \mathrm{~m} l$ dieser Lösung hinzugegeben. Die Probe wird mit $1,0 \mathrm{ml}$ der Lösung b) und $5 \mathrm{~m} l$ der Lösung c) versetzt und gut geschüttelt. Man läßt das Reaktionsgemisch 20 Minuten bei Zimmertemperatur stehen. Anschließend wird 10 Minuten zentrifugiert. Die obere Phase wird abgesaugt und verworfen. Der Rückstand wird mit Methanol bis zur $5 \mathrm{ml}$-Marke überschichtet. Unmittelbat vor der photometrischen Messung, die am Beckmanphotometer bei einer Wellenlänge von $540 \mathrm{~m} \mu$, einer Spaltbreite von $0,02 \mathrm{~mm}$ und einer Schichtdicke von $1,0 \mathrm{~cm}$ vorgenommen wird, wird der Farbstoff durch Schütteln gleichmäßig gelöst. Es wird gegen Methanol als Leerwert gemessen. Aus der Eichkurve, deren Werte unter denselben Bedingungen ermittelt wựden, lậß sich die pro Ansatz vorliegende Indicanmenge ablesen. Die Tagèsausscheidung beträgt:

$$
\frac{2 \cdot \mu \mathrm{g} \cdot \text { Urinmenge }}{1000} \mathrm{mg} \text {. }
$$

\section{Bestimmung von Indican im Serum}

Reagenzien: wie oben, jedoch

b) 0,1-proz. Thymollösung

c) $0,1 \mathrm{~g} \mathrm{FeCl}$.

\section{Ausführung}

Sic ist nahezu die gleiche wie bei der Bestimmung in Urin mit folgenden Ausnahmen: Es werden nur $0,5 \mathrm{~m} l$ einer 0,1-proz. Thymollösung (Lösung b) und nur $3 \mathrm{ml}$ der Lösung c) hinzugefügt. Mit Methanol wird auf $3 \mathrm{ml}$ aufgefüllt. Aus der entsprechenden Eichkurve kann der Indicangehalt abgelesen werden. Die Indicankonzentration ergibt sich aus der Formel: $200 \cdot \mu \mathrm{g}=$ $\mathrm{mg} / 100 \mathrm{~m} l$.

\section{Ergebnisse}

\section{Eichkurven zur Bestimmung in Urin und Serum}

Die hier angeführten Extinktionen sind der Einfachheit halber mit 1000 multipliziert.

\begin{tabular}{cccc}
\hline & $\begin{array}{c}\mu \mathbf{g} \\
\text { Indican }\end{array}$ & $\begin{array}{c}\text { Extinktion } \\
\text { (Mittelwert) }\end{array}$ & Streubereich \\
\hline \multirow{4}{*}{ Urin } & 0 & 4 & $3-4$ \\
& 5 & 54 & $53-55$ \\
& 10 & 103 & $101-105$ \\
& 20 & 203 & $200-205$ \\
& 30 & 305 & $300-310$ \\
& 40 & 400 & $395-410$ \\
& 50 & 495 & $490-500$ \\
& 75 & 740 & $730-750$ \\
& 100 & 990 & $990-1000$ \\
& 150 & 1445 & $1425-1450$ \\
& 200 & 1900 & $1900-1900$ \\
& 0 & 4 & $3-4$ \\
& 1 & 19 & $19-19$ \\
& 2 & 34 & $33-35$ \\
& 3 & 50 & $50-51$ \\
& 4 & 66 & $65-67$ \\
& 5 & 82 & $81-82$ \\
& 6 & 98 & $97-99$ \\
& 7 & 113 & $113-113$ \\
& 8 & 129 & $128-131$ \\
& 9 & 144 & $143-145$ \\
& 10 & 159 & $156-163$ \\
& 15 & 237 & $234-241$ \\
& 20 & 307 & $306-309$ \\
\hline
\end{tabular}

Die Mittelwerte wurden für Urin aus einer Vierfachbestimmung, für Serum aus einer Dreifachbestimmung berechnet.

\section{Reproduzierbarkeit}

Urin

Eine Zehnfachbestimmung mit je $0,5 \mathrm{ml}$ Urin eines Gesunden ergab folgende Extinktionen : 127/127/124/124 124/123/128/127/126/127. Mittelwert der Extinktionen mit mittlerem Fehler $(\sigma): 125,7 \pm 1,8$ oder $\pm 1,4 \%$. Das entspricht einem Indicangehalt von $12 \mu \mathrm{g} \pm 0,2 \mu \mathrm{g}$ pro Ansatz. $\mathrm{Zu}$ jeweils $0,5 \mathrm{ml}$ des gleichen Harns wurden $20 \mu \mathrm{g}$ und $50 \mu \mathrm{g}$ Indican zugesetzt. Über die Wiederauffindung gibt die folgende Tabelle Auskunft:

\begin{tabular}{|c|c|c|c|}
\hline \multicolumn{2}{|c|}{$\begin{array}{c}0,5 \mathrm{~m} / \text { Harn }+20 \mu \mathrm{g} \text { Indican }= \\
32 \mu \mathrm{g} \text { Gesamtindican }\end{array}$} & \multicolumn{2}{|c|}{$\begin{array}{c}0,5 \mathrm{~m} / \text { Ham }+50 \mu \mathrm{g} \text { Indican }= \\
62 \mu \mathrm{g} \text { Gesamtindican }\end{array}$} \\
\hline $\begin{array}{l}\mu \mathrm{g} \text { gefundenes } \\
\text { Indican }\end{array}$ & $\%$ Fehler & $\begin{array}{l}\mu \mathrm{g} \text { gefundenes } \\
\text { Indican }\end{array}$ & $\%$ Fehler \\
\hline $\begin{array}{l}32,5 \\
32,5 \\
31,0 \\
31,0 \\
31,5\end{array}$ & $\begin{array}{l}+1,6 \\
+1,6 \\
-3,1 \\
-3,1 \\
-1,6\end{array}$ & $\begin{array}{l}60,5 \\
59,5 \\
60,5 \\
60,5 \\
60,5 \\
60,5\end{array}$ & $\begin{array}{l}-2,4 \\
-4 \\
-2 \\
-2 \\
-2 \\
-2\end{array}$ \\
\hline
\end{tabular}




\section{Serum}

Eine Vierfachbestimmung mit je $0,5 \mathrm{ml}$ Serum ergab eine konstante Extinktion von 12, das sind 0,5 $\mu \mathrm{g}$. Die Ergebnisse nach Zusatz von 5 bzw. $10 \mu \mathrm{g}$ Indican sind in der folgenden Tabelle zusammengestellt:

\begin{tabular}{|c|c|c|c|}
\hline \multicolumn{2}{|c|}{$\begin{array}{c}0,5 \mathrm{~m} / \text { Serum }+5 \mu \mathrm{g} \text { Indican }= \\
5,5 \mu \mathrm{g} \text { Gesamtindican }\end{array}$} & \multicolumn{2}{|c|}{$\begin{array}{c}0,5 \mathrm{~m} / \text { Serum }+10 \mu \mathrm{g} \text { Indican } \\
10,5 \mu \mathrm{g} \text { Gesamtindican }\end{array}$} \\
\hline $\begin{array}{l}\mu \mathrm{g} \text { gefundenes } \\
\text { Indican }\end{array}$ & $\%$ Fehler & $\begin{array}{l}\mu \mathrm{g} \text { gefundenes } \\
\text { Indican }\end{array}$ & $\%$ Fehler \\
\hline 5,3 & $-3,6$ & 10,0 & $-4,8$ \\
\hline 5,4 & $-1,8$ & 10,0 & $-4,8$ \\
\hline 5,0 & $-9,1$ & 9,9 & $-5,7$ \\
\hline 5,3 & $-3,6$ & 10,0 & $-4,8$ \\
\hline 5,1 & $-7,3$ & 10,1 & $-3,8$ \\
\hline 5,3 & $-3,6$ & 9,9 & $-5,7$ \\
\hline
\end{tabular}

Der Verlust tritt wahrscheinlich bei der Eiweißfällung ein.

\section{Empfindlicbkeit}

Die Methode erlaubt die Auffindung von $0,1 \mu \mathrm{g}$ im Serum. Die Genauigkeit der Bestimmung bei niedriger Indicankonzentration im Serum kann ohne weiteres durch die doppelte Ausgangsmenge $(1 \mathrm{~m} /)$ gesteigert werden.

\section{Spezifität}

Die Übereinstimmung der Absorptionsmaxima in Abbildung 1 und Abbildung 2 zeigt, daß der bei der Indicanbestimmung im Urin oder Serum entstehende Farbstoff dem Kondensationsprodukt von Indican und Thymol entspricht. Über das Verhältnis der eventuell störenden Indolderivate gibt die Abbildung 3 Auskunft.

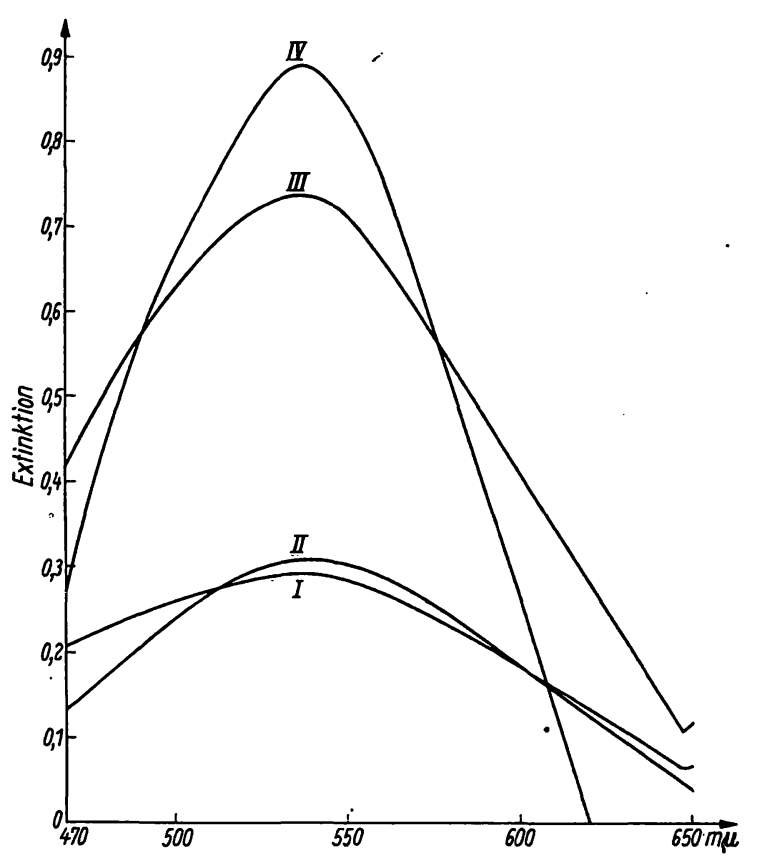

Abb. 1

I Urin eines Patienten mit Diabetes mellitus, II Indican, III Urin eines Patienten mit Verschlußikterus, IV Urin eines Patienten mit Lebercirrhose (im Verhältnis 1:2)

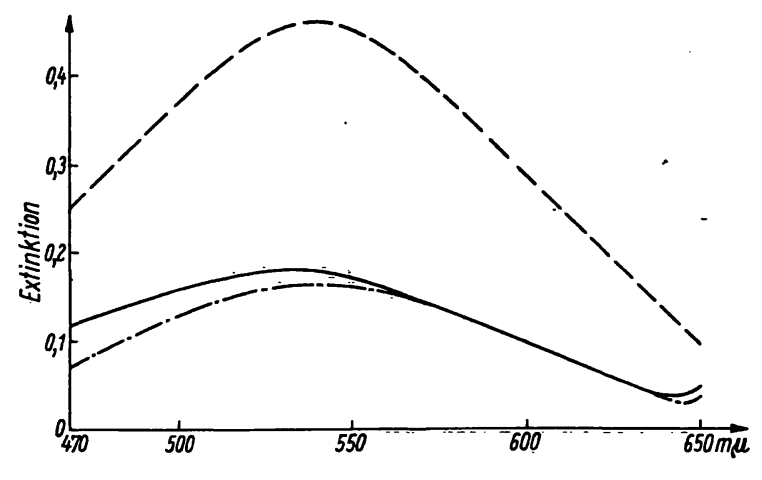

Abb. 2

Mittlere Kurve Indican, die beiden anderen Kurven Seren von Patienten mit Niereninsuffizienz

Von elf getesteten Indolderivaten ergab Indoxylacetat (V) ebenso wie Indican (VI) einen rotvioletten Farbstoff, da es durch Hydrolyse ebenfalls in Indoxyl überführt wird. Dieser liegt in seiner Intensität im Bereich der durch Anwesenheit der gleichen Menge Indican hervorgerufenen Farbe. Indolessigsäure (I), 5-Hydroxyindolessigsäure, Skatol (III), Indol (II) und 5-Hydroxyindol (IV) zeigen eine Farbentwicklung, deren Extinktion unter 10\% der entsprechenden Indicanmenge liegt, während Indolbuttersäure, Indolpropionsäure, Indolmilchsäure, Serotonin und Tryptophan keine Farbreaktion bewirken. Wie die papierchromatographischen Ergebnisse von JEPSON (4) und auch eigene Untersuchungen zeigen, ist die Konzentration der eventuell störenden Indolderivate in Urin und Serum selbst bei schwerer Niereninsuffizienz so gering, daß der Nachweis von Indican nicht gestört wird (5). Bilirubin, das durch Oxydation das grüne Biliverdin bildet, zeigt unter den

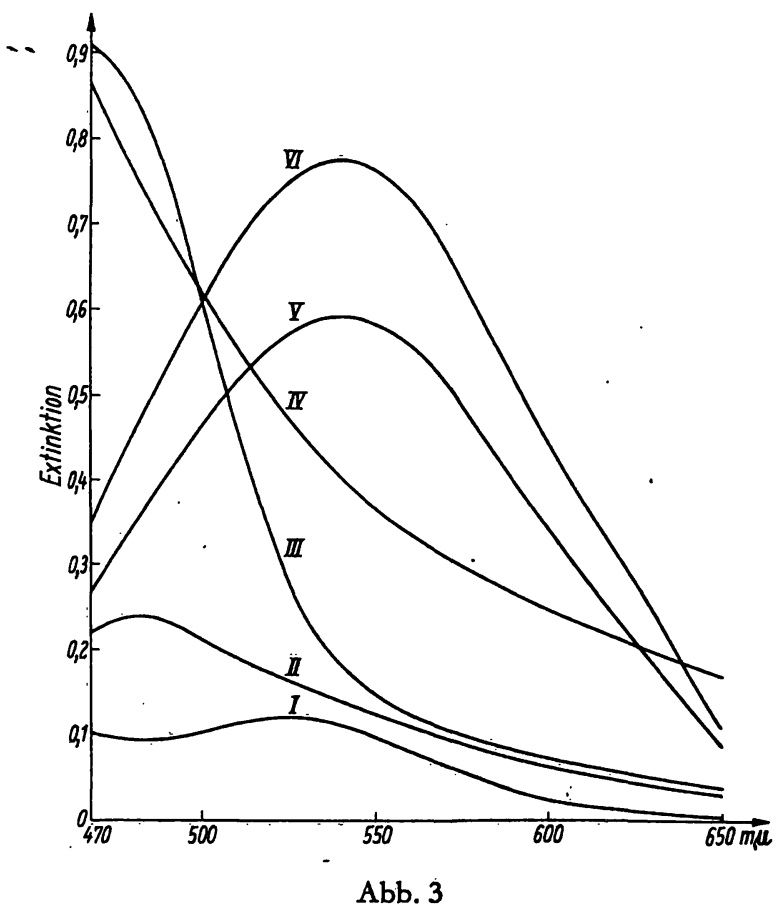

Indolderivate, die eine Färbung ergaben. I Indolessigsäure $(500 \mu \mathrm{g})$, II Indol $(500 \mu \mathrm{g})$, III Skatol $(500 \mu \mathrm{g})$, IV 5-Hydroxyindol $(500 \mu \mathrm{g})$, V Indoxylacetat $(60 \mu \mathrm{g})$, VI Indican $(80 \mu \mathrm{g})$ 
Bedingungen der Bestimmungsmethode eine Farbintensität, die ungefähr $25 \%$ einer gleichkonzentrierten Indicanlösung ausmacht, was als Deutung der grünen Farbe bei Untersuchungen des Urins einiger Leberkranker angeführt werden kann.

\section{Haltbarkeit der Farbe}

Nach Lösen in Methanol nimmt die Farbintensität innerhalb 30 Minuten um rund $5 \%$ ab. Es soll deshalb sofort nach dem Lösen abgelesen werden.

\title{
Literatur
}

1. Jolles, A., Hoppe-Seyler's Z. physiol. Chem. 94, 79 (1915). 2. RAppaport, F. und H. Engelberg, Klin. Wschr. 12, 71 (1933). - 3. Shivaram, K. N. und D. Müring, Aerztl. Forschg., Wöris- hofen 15, I/337 (1961). - 4. Jerson, J. B., in: I. Smith, Chromatographic and Electrophoretic Techniques 1, 183 (1960). - 5. Müring, D., Gastroenterologia (Basel), im Druck.

Professor Dr. D. Müting, 1. Medizinische Universitätsklinik, 665 Homburg (Saar)

\section{Separation of Cystine-Amino-Peptidase and Leucine-Amino-Peptidase and their determination in pregnant and nonpregnant women}

By U. GoebelsmanN ${ }^{1}$ ) and F. K. BelleR ${ }^{2}$ )

From the Department of Obstetrics and Gynecology, Nenע York University School of Medicine, Nenע York, US $A$

(Der Schriftleitung zugegangen am 26. August 1964)

\begin{abstract}
Serum leucine-aminopeptidase (LAP) and cystine-aminopeptidase (CAP) determinations have been made using a chemical method based on enzymatic hydrolysis of 1-leucine- $\beta$-naphthylamide-hydrochloride and cystine-di- $\beta$ naphthylamide, respectively. Fractionation of serum proteins by gel filtration on "Sephadex-G 200 " has been used for separation of LAP and CAP (oxytocinase). - CAP which appears during pregnancy only and LAP present in all sera, hydrolyse both substrates, LNA and CDNA. LAP has been found to cleave LNA 74 times faster than CDNA and CAP has been confirmed to hydrolyse LNA 11 times faster than CDNA.

CAP-activity found in non-pregnant serum with small, but significant values, is due to LAP hydrolysing CDNA. Comparing these CAP-activity values in normal individuals with CAP-activity levels at term, only a 13-fold increase of CAP-activity can be recovered. Subtraction of CAP-activity due to LAP from total CAP-activity in early pregnancy reveals against net CAP values at term an increase of CAP activity which is comparable to ratios found with oxytocinase determinations based on a bioassay methods. - Separation of CAP from LAP on "Sephadex" gel dilutes small enzyme concentrations as appear during the first half of gestation to such an extent as to make pooling and concentration procedures necessary in order to achieve appreciable accuracy for CAP assays.

The slow increase of CAP during the first half of gestation, the variation of LAP-activity in normal individuals and the mutual substrate unspecificity of both enzyme render LAP and CAP assays invalid for the diagnosis of early pregnancy.
\end{abstract}

Bestimmungen von Serum-Leuzin-Aminopeptidase (LAP) und Cystin-Aminopeptidase (CAP) wurden mit Hilfe eines chemischen Verfahrens vorgenommen, das auf der enzymatischen Hydrolyse von l-Leuzin- $\beta$-naphthylamidhydrochlorid und entsprechend Cystin-di- $\beta$-naphthylamid beruht. Zur Trennung von LAP und CAP (Oxytocinase) wurde die Fraktionierung der Serumproteine mit Hilfe von Gelfiltration an "Sephadex-G 200" verwendet. CAP, welches nur während der Gravidität exscheint, und LAP, das in allen Seren vorhanden ist, hydrolysieren sowohl LNA wie CDNA. Es wutde gefunden, daß LAP LNA 74mal schneller aufspaltet als CDNA und CAP hydrolysiert LNA $11 \mathrm{mal}$ schneller als CDNA.

CAP-Aktivität, welche mit geringen aber eindeutigen Werten im Serum von Nichtgraviden nachgewiesen wird, beruht auf LAP, welche CDNA hydrolysiert. Vergleicht man diese CAP-Aktivitätswerte bei normalen Individuen mit CAP-Aktivitätswerten am Termin, so kann nur ein 13faches Ansteigen der CAP-Aktivität festgestellt werden. Zieht man die durch LAP verursachte CAP-Aktivität vom Gesamt-CAP-Aktivitätswert bei Schwangerschaftsbeginn ab, so zeigt sich gegen reine CAP-Werte am Termin eine Zunahme der CAP-Aktivtät, welche vergleichbar ist den Werten, die mit Oxytocinase-Bestimmungen gefunden wurden, die auf biologischer Methodik beruhen. Trennung der CAP von LAP an "Sephadex-gel" verdünnt kleine Enzymkonzentrationen, wie sie während der ersten Hälfte der Schwagerschaft erscheinen, in einem solchen Ausmaß, daß Sammeln und Konzentrierungsmaßnahmen notwendig sind, um wirkliche Zuverlässigkeit für CAP-Bestimmungen zu erhalten. Die langsame Zunahme von CAP während der exsten Hälfte der Schwangerschaft, die Streuung der LAP-Aktivität bei gesunden Individuen und die wechselseitige Substrat-Unspezifität beider Enzyme machen LAP- und CAP-Bestimmungen für die Frühdiagnose der Schwangerschaft ungeeignet.

The inactivation of oxytocin by human and rhesus monkey plasma during pregnancy has been attributed

1) Trainee, United States Public Health Service (2 C 400).

2) Career Scientist of the Health Research Council of the City of New York (I-297). to an enzyme called oxytocinase (1). Studies on oxytocinase have been extended by several groups of investigators $(2-5)$. In the above studies the amount of oxytocin not inactivated was determined subsequently by bioassays. 